\title{
ROADMAPPING ÁGIL: APLICAÇÃO DO ROADMAPPING INTEGRADO AO SCRUM COMO SUPORTE AO PLANEJAMENTO CONTÍNUO EM UMA EMPRESA JR
}

Carolina Campos Nagem Araújo (nagemcarolina@gmail.com) - Pontifícia Universidade Católica de Minas Gerais - PUC Minas

Fernanda Llore Martins Lana (nandallore@gmail.com) - Pontifícia Universidade Católica de Minas Gerais - PUC Minas

Jorge de Oliveira Costa Pereira Neto (jorgedo1998@gmail.com) - Pontifícia Universidade Católica de Minas Gerais - PUC Minas

Paola Vianelo (paolavianello10@ hotmail.com) - Pontifícia Universidade Católica de Minas Gerais PUC Minas

Matheus Luiz Pontelo de Souza (mslzposa@gmail.com) - Pontifícia Universidade Católica de Minas Gerais - PUC Minas

\section{RESUMO}

O método Roadmapping se consolidou mundialmente como forma de apoio ao planejamento estratégico em empresas, fomentando a integração entre visão de futuro e ação no presente de forma organizada e coletiva. O método SCRUM, por sua vez, atua na gestão de projetos, com flexibilidade e agilidade, com foco na orientação de times pequenos tendo por base reuniões frequentes, integração entre colaboradores e entregues visíveis. Entretanto, apesar da grande utilização do roadmapping, há desafios quanto à aplicação em pequenas empresas/empresas nascentes. Um dos fatores que compõem tais desafios é a necessidade de atualização contínua do mesmo. O estudo teve por base a pesquisa ação em uma parceria entre um grupo de pesquisa e uma empresa Jr. da Engenharia de Produção. O objetivo do estudo foi descrever a aplicação integrada do roadmapping com o SCRUM. Para tanto, foi descrito o processo de construção de um roadmapping de longo prazo, bem como uma conexão de tal processo com o SCRUM. A aplicação foi útil para ajudar na formulação estratégia, bem como em seu desdobramento operacional com atualizações rápidas e rotineiras. O método integrado se mostrou útil para 


\section{Congresso Brasileiro de Inovação e Gestão}

de Desenvolvimento do Produto

11 a 13 de setembro de 2019 - Universidade de Brasília UnB

criação de um planejamento ágil e flexível, demonstrando potencial frente à necessidade de pequenas empresas. 


\section{2 - Congresso Brasileiro de Inovação e Gestão}

\section{de Desenvolvimento do Produto}

\section{1 a 13 de setembro de 2019 - Universidade de Brasília UnB}

\section{INTRODUÇÃO}

O método Roadmapping se consolidou mundialmente como forma de apoio ao planejamento estratégico conjunto, em empresas dos mais diversos setores (Phaal et al. 2010). O roadmapping é um método que utiliza de um quadro para auxiliar no planejamento tático de forma rápida, coletiva e com objetivo de auxiliar na integração da visão estratégica do futuro com as ações do presente.

Foi destacado nesse método alguns problemas voltados à aplicação em pequenas empresas e à sua atualização contínua (Gerdsri et al., 2009). As dificuldades para aplicação estão relacionadas à adaptação do método às especificidades das pequenas empresas (e.g.: conhecimento sobre a aplicação de métodos, baixa disponibilidade de recursos) e à atualização contínua do roadmap e planos de ação gerados pelo esforço de construção do roadmapping.

Alguns autores, como Souza et al (2018) e Carlos et al. (2018), têm utilizado com sucesso a integração de roadmapping a métodos da escola ágil de projetos, de modo a sanar os desafios citados acima. Também é conhecido que métodos como o SCRUM são úteis para gestão de times pequenos, o que motivou sua introdução para auxiliar na gestão do plano estratégico ao operacional, passando pelo tático, de uma empresa júnior, que tem necessidade de agilidade, com atualizações rápidas do planejamento estratégico.

Logo, o objetivo deste estudo foi aplicar conjuntamente os dois métodos como forma de auxiliar à formulação de um planejamento estratégico em horizonte de tempo próximo ao anual, bem como ajudar no acompanhamento dos planos de ação na rotina da empresa Jr. via mecanismos de atualizações rápidas e rotineiras. $\mathrm{O}$ estudo se propõe a também fornecer indicações que possam auxiliar pequenas empresas de modo semelhante.

A abordagem escolhida foi de pesquisa-ação, por meio da qual o grupo de pesquisa GETI-Praça da Liberdade auxiliou a Easy Consultoria Jr. na criação de um processo de roadmapping, que resultou em um planejamento de um ano e a aplicação do SCRUM para operacionalizar as definições estratégicas. 


\section{2 - Congresso Brasileiro de Inovação e Gestão}

de Desenvolvimento do Produto

11 a 13 de setembro de 2019 - Universidade de Brasília UnB

Como resultado tivemos um processo de roadmapping adaptado e integrado ao SCRUM, gerando resultados na área de planejamento da empresa Jr. com a facilidade e agilidade na atualização e revisão de metas e tarefas. Implicações gerenciais foram levantadas a partir da aplicação, implementação e distribuição das tarefas no planejamento, com auxílio do roadmap.

\section{REVISÃO TEÓRICA}

Será exposto nesse tópico, o referencial teórico do roadmapping e do SCRUM, exemplificando as bases teóricas utilizadas para subsidiar o desenvolvimento da pesquisa e aplicações, contidas no artigo.

\subsection{Roadmapping}

O roadmapping é definido por Phaal et al (2010) como um método composto de uma estrutura visual que é usada para criação de um roteiro para dar suporte à processos de inovação e estratégia. Freitas et al (2017) ressaltam que o método é usado por conta da alta capacidade de aplicabilidade e flexibilidade na integração dos níveis dos processos de negócios.

A estrutura do roadmap, ilustrada na Figura 1, pode ser diferenciado por conta da sua estrutura baseada em dois eixos principais, o eixo x e eixo y. O eixo x representa o horizonte temporal no presente, médio e longo prazo e o eixo y representa a arquitetura do mapa expressa em termos de camadas necessárias para o propósito da aplicação do método, como mercados/negócios e produtos/serviços (PHAAL; MULLER, 2009). 


\section{2 - Congresso Brasileiro de Inovação e Gestão}

\section{de Desenvolvimento do Produto}

11 a 13 de setembro de 2019 - Universidade de Brasília UnB

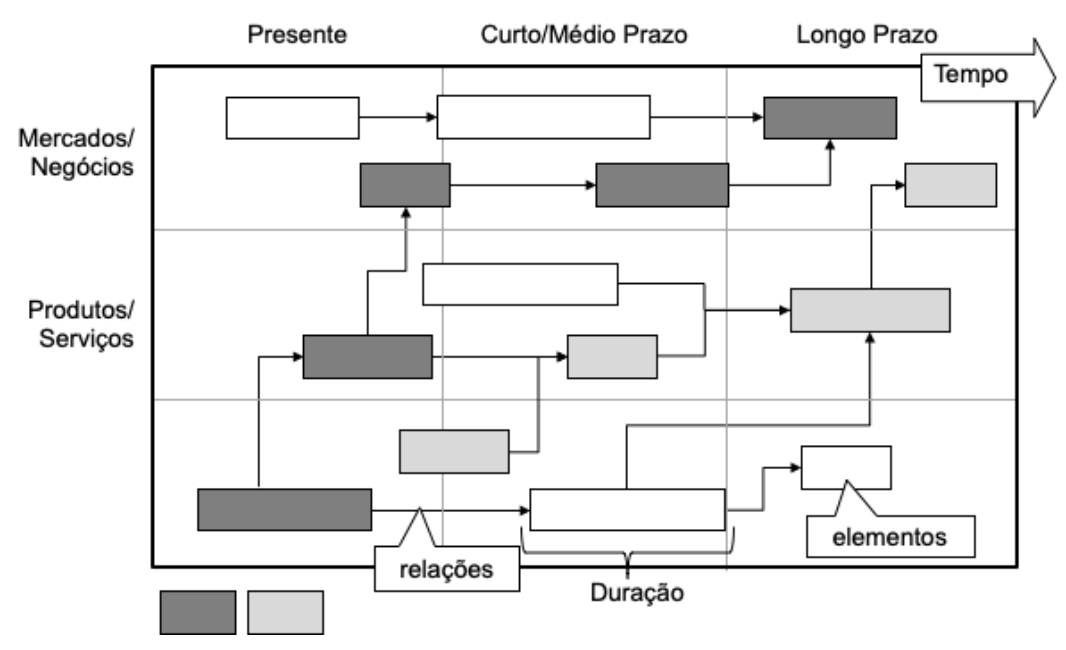

Fonte: Oliveira et al (2012)

Este estudo se baseou na abordagem rápida descrita pelo T-Plan (PHAAL; FARRUKH; PROBERT, 2013). A Figura 2 ilustra o processo de aplicação do T-Plan e sua divisão em seminários (workshops), anteposto de um momento de planejamento (OLIVEIRA el al, 2012). Nesse momento de planejamento é definido qual será a estrutura do roadmap, definindo o conteúdo das camadas e subcamadas do mapa, os participantes e recursos existentes, a demanda, dentre outros. Posteriormente ao planejamento, é fundamental a realização de seminários para personalização do método, com o intuito de dedicar-se nas questões de mercado, produto, tecnologia e construção do mapa em si. Consiste também na estratégia de organização rápida, mas se apoiando o planejamento de produtos e serviços, com foco em níveis mais amplos (PHAAL et al, 2001).

Figura 2: Roadmapping para nível de produto/Tecnologia

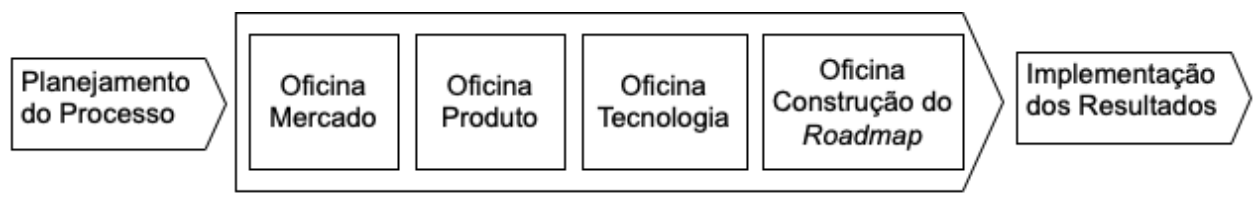

Fonte: Oliveira et al (2012)

\subsection{SCRUM}

O SCRUM é um método da escola ágil de gestão de projetos e apresenta características de flexibilidade, agilidade, e orientação do trabalho de times pequenos. Esse método realiza reuniões frequentes, com a participação de todos, para obtenção do melhor resultado possível. 


\section{2 - Congresso Brasileiro de Inovação e Gestão}

de Desenvolvimento do Produto

\section{1 a 13 de setembro de 2019 - Universidade de Brasília UnB}

O modo de trabalho desse método é realizado com um desdobramento da lista de funções, durante o projeto, chamada de backlog do produto, como mostrado na figura 3 . O desdobramento é baseado em sprints, que são intervalos de tempos de uma a quatro semanas, que no final gera um entregue visível (SCHWABER, 2004). Segundo Schwaber (2004) os backlogS podem ser definidos como uma lista de requisitos funcionais e não funcionais, que após desenvolvimento, darão a visão inicial da empresa.

Figura 3: Visão geral do processo SCRUM

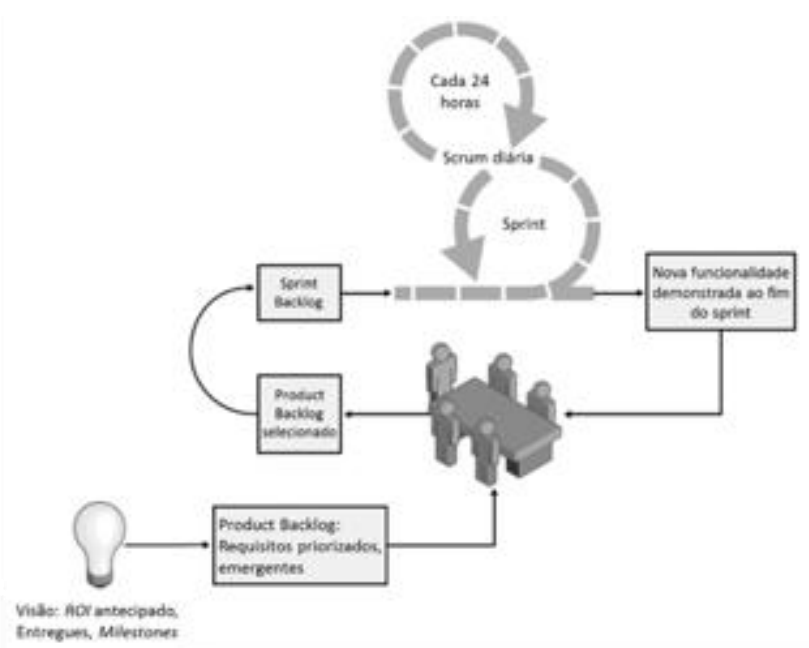

Fonte: Schwaber (2004)

Schwaber (2004) define a seleção de backlog do sprint, na qual a equipe realiza reuniões diárias, com objetivo de conduzir o ritmo de trabalho, facilitando na comunicação de tarefas realizadas no dia anterior, por cada membro. Com isso, ajudando na decisão das tarefas do dia atual e abordagem dos possíveis empecilhos para realização. Criando assim uma importante função, o SCRUM master. No final de cada sprints são entregues resultados visíveis, em uma reunião de avaliação do sprints atual e planejamento do próximo a ser realizado.

\section{METODOLOGIA}

A abordagem de pesquisa utilizada foi a pesquisa-ação (COUGHLAN; COGHLAN, 2002; THIOLLENT, 2009), visto que o objetivo era aplicar e integrar os métodos Roadmapping e SCRUM em uma empresa jr. e acompanhar tanto a aplicação quanto os resultados que ela 


\section{2 - Congresso Brasileiro de Inovação e Gestão}

\section{de Desenvolvimento do Produto}

11 a 13 de setembro de 2019 - Universidade de Brasília UnB

poderia gerar em termos teóricos e práticos. Conforme dizem os autores, a pesquisa-ação é mais pesquisa em ação do que sobre a ação, na qual pesquisadores e detentores do problema interagem de forma real para atingir os objetivos propostos. Neste caso, o problema de planejamento estratégico, tático e operacional em uma empresa Jr. nascente.

A pesquisa ação foi realizada na PUC-Minas, unidade Praça da Liberdade, Departamento de Engenharia de Produção. A aplicação do roadmapping integrado ao SCRUM foi feita na empresa Jr. denominada Easy consultoria júnior, fundada na PUC-Minas, unidade Praça da Liberdade. Foram diretamente envolvidos na aplicação, da parte da empresa Jr., a presidente, o diretor comercial e a diretora de projetos. Em um momento ao longo do processo de roadmapping, os demais membros da Easy foram também envolvidos, embora de modo pontual. A aplicação foi apoiada pelo grupo de pesquisa GETI (Grupo de Empreendedorismo Tecnológico e Inovação), pertencente à mesma unidade e departamento da empresa Jr. Da parte do grupo de pesquisa, quatro pesquisadores foram envolvidos, bem como o professor coordenador do mesmo.

A pesquisa se baseou na montagem de um Roadmap integrado ao SCRUM, com o objetivo de aplicação desses métodos no contexto da Easy Consultoria Jr. e acompanhamento de todo o processo, tanto de criação, quanto de aplicação e análise. O processo foi iniciado em março/2019 e segue até o momento de escrita deste artigo. Foram realizadas 5 reuniões formais (em formato próximo ao de workshops), com uma hora de duração em média. Outras reuniões menores, bem como comunicação informal física e via outros meios como Trello e WhatsApp também foram utilizados durante a aplicação.

\section{RESULTADOS E DISCUSSÃO}

Nessa parte do artigo, as partes de desenvolvimento do roadmapping, do SCRUM e a aplicação conjunta dos dois métodos será detalhada com mais clareza. Logo após, uma análise crítica sobre os métodos utilizados e a maneira com que eles foram aplicados será exposta, para que possa ser analisado o que deveria ter sido feito diferente e quais pontos foram positivos dessa experiência, além dos resultados para a empresa júnior em questão.

\subsection{A aplicação conjunta dos métodos}




\section{2 - Congresso Brasileiro de Inovação e Gestão}

\section{de Desenvolvimento do Produto}

\subsubsection{O processo de Roadmapping}

O roadmapping foi construído ao longo de cinco reuniões formais para discorrer sobre os temas mercado, produto/serviços e recursos, sendo a construção do mapa feita de modo contínuo (Figura 4). A oficina de mercado ocorreu em duas reuniões para definição de metas e ações específicas, a oficina de produto definiu quais os produtos e/ou serviços que a empresa realizaria para atingir as metas e, finalmente, a oficina de recursos definiu tanto os novos processos que a empresa Jr. deveria implementar quanto os recursos que seriam necessários para implementar as definições traçadas em todo o roadmap. Uma reunião adicional de brainstorming com possibilidades de enriquecimento do mapa foi feita. A construção do roadmapping foi realizada em paralelo com as reuniões citadas. A Figura 4 detalha o processo de roadmapping utilizado.

Figura 4: Descrição do Processo Integrado de Roadmapping-SCRUM Utilizado

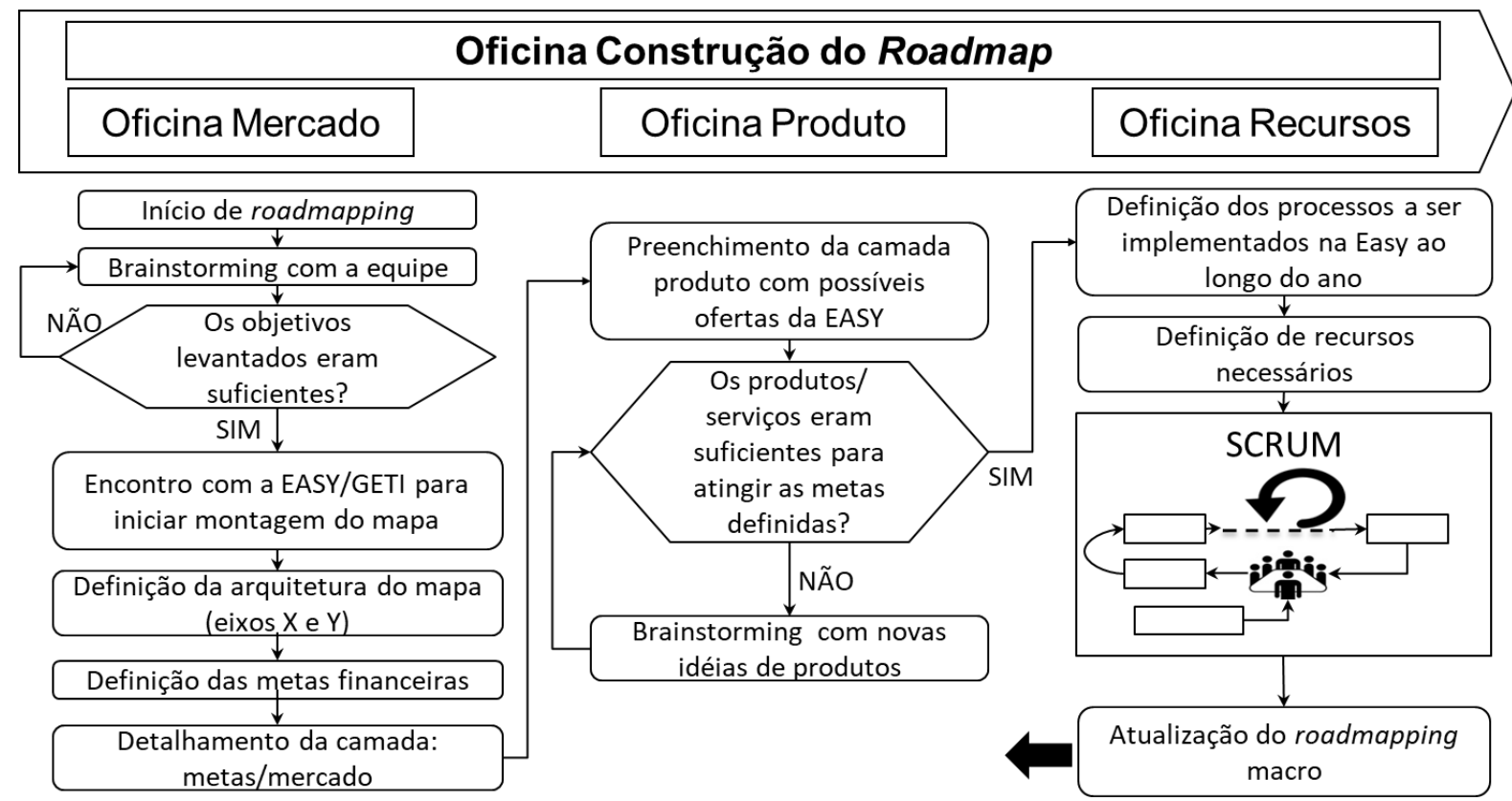

Fonte: Elaborado pelos autores

Para início do trabalho de estruturação do roadmapping para a empresa Jr foi realizada uma reunião com o objetivo de definir as necessidades da empresa Jr. ao longo do ano, expressas em termos financeiros. Para isso, foram feitas análises e consultas a fornecedores, antes da reunião, com o intuito de esboçar um fluxo de caixa anual. Este fluxo foi analisado durante a 


\section{2 - Congresso Brasileiro de Inovação e Gestão}

\section{de Desenvolvimento do Produto}

\section{1 a 13 de setembro de 2019 - Universidade de Brasília UnB}

reunião e, com base nele, foram definidas metas financeiras desdobradas ao longo do ano. Estas metas foram o input macro da camada de mercado.

Em seguida, fez-se uma reunião com toda a equipe da Easy Consultoria Jr. para levantar objetivos e ações gerais para a empresa, sem prazo definido. Em formato de brainstorming, os objetivos/ações foram levantados e discutidos e, caso não considerados realizáveis, novas ideias ou adaptações foram pensadas. Depois de anotados todos os objetivos e ideias, foram levados para as próximas reuniões de roadmapping, incluindo a direção da Easy Consultoria Jr. e os membros do GETI. O brainstorming resultante dessa reunião foi útil como forma de preenchimento de gaps e estímulo à criatividade mediante proposição de novas ideias durante todo o processo do roadmapping.

Em sequência, foi realizada uma reunião para montar o esboço inicial do mapa, começando pela definição da arquitetura do mesmo. O horizonte de tempo definido foi de um ano, visto que planejamentos de longo prazo seriam imprecisos no contexto. Foram especificados períodos de tempo de dois meses no eixo X até atingir o início do ano de 2020, de modo a facilitar a comunicação bimestral das ações expostas no mapa com a operação do SCRUM.

As camadas definidas foram metas/mercado, produto/serviços e recursos/processos, de modo a simplificar a compreensão do mapa em termos do Por Quê (Why), O quê (What) e Como (How). Após definir a arquitetura do mapa, filtraram-se os objetivos levantados na reunião anterior, com a intenção de transformá-los em ações de mercado mais práticas e realizáveis. Com essas metas analisadas e definidas, a camada superior "metas/mercado" foi preenchida e detalhada.

Em seguida, iniciou-se a definição da camada "produtos/serviços" com base na análise da primeira camada da tabela. Nesta camada foram assinaladas ofertas da empresa Jr. como cursos, projetos de consultoria com clientes e venda de brindes. Essa tarefa foi iniciada na mesma reunião na qual se definiu a primeira camada do mapa e concluída posteriormente. Durante a primeira reunião, participaram a diretoria da empresa, os membros do GETI e o professor orientador, que atuou como facilitador neutro (KERR et al, 2013) durante o processo. Iniciandose com uma breve aula sobre o tema Roadmapping e prosseguindo com a montagem do mapa 


\section{2 - Congresso Brasileiro de Inovação e Gestão}

\section{de Desenvolvimento do Produto}

\section{1 a 13 de setembro de 2019 - Universidade de Brasília UnB}

e estratetização coletiva. Neste momento, o facilitador pode ensinar um pouco sobre o que seria feito dali para frente, mas sem especificar muito, o que será analisado na seção 4.2.

Os gaps evidenciados por essa reunião mostraram que a empresa precisava criar novas ideias de projetos de produto/serviço, começar a trabalhar com clientes, agilizar alguns processos jurídicos e começar a se preocupar, não apenas com o que ganhar, mas como ganhar, isto é, com a estruturação de processos e mobilização de recursos humanos/financeiros. Além disso, foi evidenciada a necessidade de distribuir as tarefas de maneira justa para todos da empresa já que os membros da diretoria estavam sobrecarregados, enquanto os outros membros da equipe não estavam participando ativamente. Para este último aspecto, a implantação posterior do SCRUM foi útil e tem sido acompanhada neste esforço de pesquisa.

Se os produtos e serviços definidos eram o bastante para alcançar as metas de mercado estabelecidas, seguia-se para o próximo passo (Figura 4). Caso contrário, elas deveriam ser revistas e reanalisadas para que cumprissem com os objetivos da camada posterior, ou até mesmo realizado um novo brainstorming para elaboração de novos produtos ou serviços. Esta reflexão foi conduzida predominantemente por vias de comunicação informal presencial ou suportada por meios digitais.

Em seguida, foi realizada outra reunião para a definição da camada recursos/processos, dessa vez apenas com a direção da Empresa Jr. Com a duração de, em média, 30 minutos, foi possível descrever o que seria preciso realizar na prática e quais recursos seriam necessários para isso, mas a falta de conhecimento teórico sobre a aplicação gerou insegurança na equipe e pode ter trazido dificuldades para o desenvolvimento, uma vez que nesta reunião o facilitador não estava presente. Surgiu, então, dúvida em relação aos recursos, que foi esclarecida posteriormente com o facilitador e, então, resolveu-se tirá-la do mapa, por falta de necessidade.

Com as três camadas do mapa (roadmap) completas e analisadas, ele foi redesenhado digitalmente para melhor visualização, utilizando o Powerpoint, diferenciando tarefas por cores e localizando-as em seus determinados lugares na tabela. A Figura 5 demonstra o roadmap construído. O mapa construído mostrou escassez de definições abaixo e à direita, o que foi feito propositalmente de modo a incorporar o princípio da gestão ágil de projetos segundo o qual 


\section{2 - Congresso Brasileiro de Inovação e Gestão}

de Desenvolvimento do Produto

CONGRESSO BRASILEIRO DE INOVAC̆ÃO E GESTÁO DE DESENYOLVIMENTO DO PRODUTO

11 a 13 de setembro de 2019 - Universidade de Brasília UnB

deve-se desdobrar as ações em maiores de detalhamento apenas quando estas ações estão próximas de ocorrer.

Figura 5: Roadmap resultante

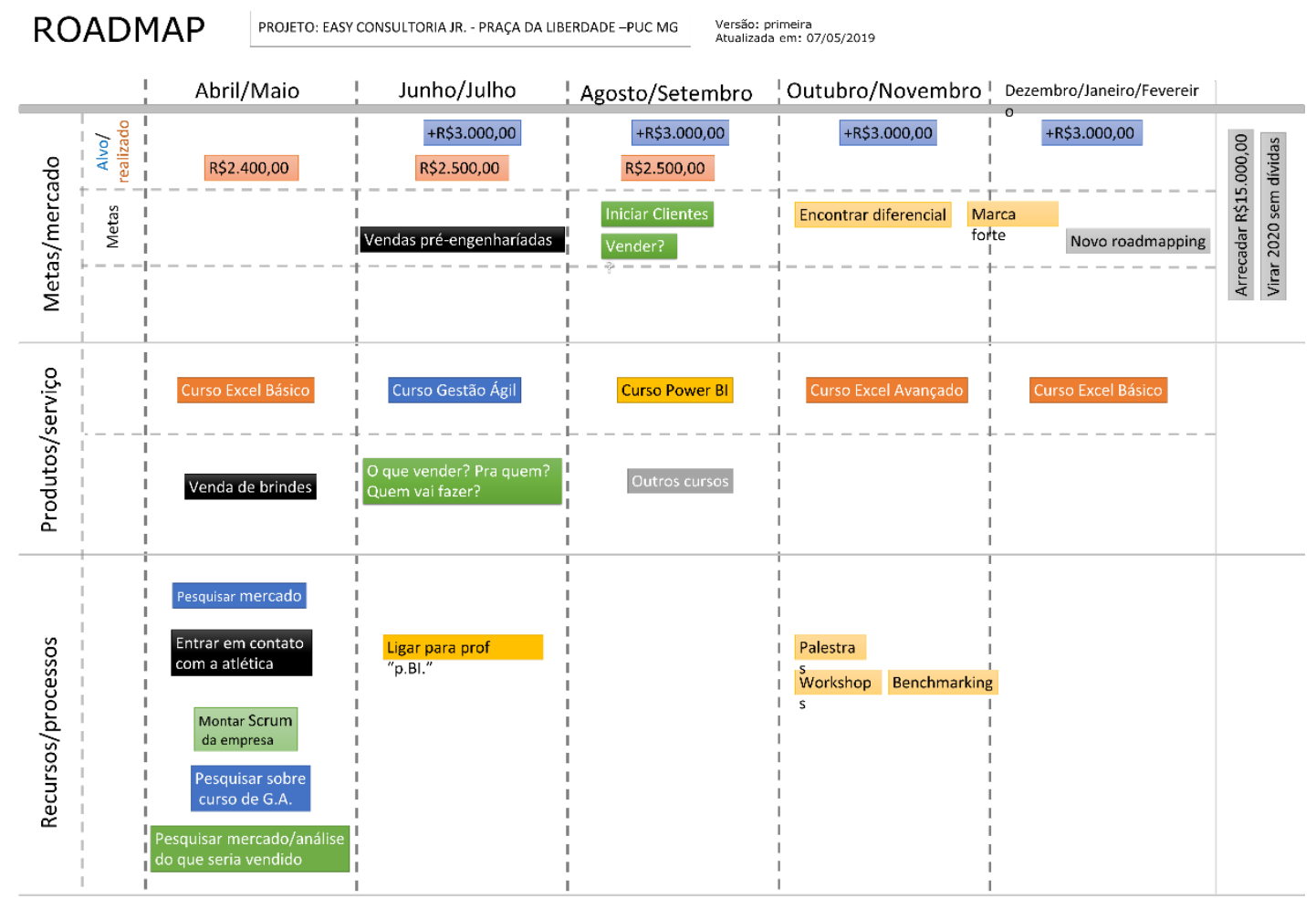

Fonte: Elaborado pelos autores

\subsubsection{Implementação do SCRUM}

Em uma última reunião de adaptação dos métodos, com os participantes da empresa Jr., GETI e o facilitador, foram definidas as rotinas e rituais que se seguiriam durante a utilização do SCRUM. O ciclo do SCRUM se deu conforme a figura 6 e será abaixo detalhado. A aplicação inicial, embora acompanhada por pouco tempo durante essa pesquisa, demonstrou resultados satisfatórios para a equipe da empresa Jr.

Figura 6: Visão Geral do Processo SCRUM 


\section{2을 Congresso Brasileiro de Inovação e Gestão}

\section{de Desenvolvimento do Produto}

11 a 13 de setembro de 2019 - Universidade de Brasília UnB

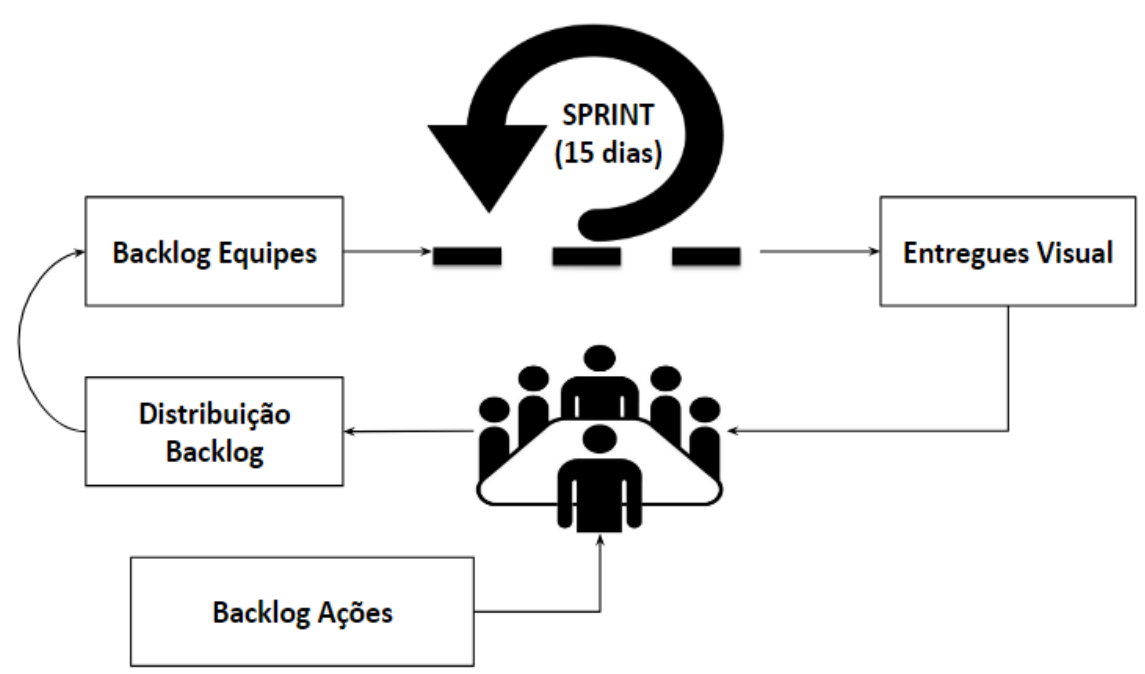

Fonte: Adaptado de Schwaber (2004)

Primeiramente, o backlog de ações (Figura 6) foi obtido a partir do fracionamento do roadmap (Figura 5) em bimestres. Cada bimestre, assim, gera um backlog como entrada do SCRUM. A distribuição do backlog (Figura 6) foi feita para os membros (ou equipes) da empresa Jr. em reunião coletiva, inicialmente acompanhada pela equipe do GETI. Assim, montou-se uma tabela no Excel (Figura 7), desdobrando cada tarefa em tarefas específicas, atribuíveis aos envolvidos, envolvendo prazos que poderiam ser executados durante um sprint (15 dias).

Figura 7: Exemplo de backlog de ações em Excel

\begin{tabular}{|c|c|c|c|c|}
\hline Referência no ROADMAP & A FAZER & SPRINT & RESPONSÁVEL & PRAZO \\
\hline \multirow[t]{3}{*}{ Venda de Brindes ATHL } & Formalizar negociação: o que vender e \% & & & \\
\hline & Mobilizar força de vendas & & & \\
\hline & Acompanhar força de vendas & & & \\
\hline \multirow[t]{2}{*}{ Venda de brindes CURSOS } & Pesquisa sobre possiveis mercados e brindes de venda & 1 & PESSOA 1 & $20 / 05$ \\
\hline & pesquisa de empresas para fazer a produção & 1 & PESSOA 2 & $25 / 05$ \\
\hline Curso XLS Básico & OK & & & \\
\hline \multirow[t]{7}{*}{ Curso gestão agil } & Pesquisa mercado: preço e aderência & 1 & PESSOA 3 & $15 / 05$ \\
\hline & Definição de custo e vagas & 1 & DIRETORIA & $20 / 05$ \\
\hline & Preparação do conteúdo & 3 & DIRETORIA & $20 / 05$ \\
\hline & Elaboração do material de divulgação & 3 & MARKETING & $21 / 05$ \\
\hline & Entregar material de divulgação completo & 3 & MARKETING & $30 / 05$ \\
\hline & Início das divulgações & 3 & TODA A EQUIPE - DEFINIR & $01 / 06$ \\
\hline & Infraestrutura & 2 & PESSOA 4 & $22 / 05$ \\
\hline
\end{tabular}

Fonte: Elaborado pelos autores

O repasse para os membros da equipe foi feito com auxílio do software Trello, no qual as tarefas repassadas ficaram armazenadas. A partir daí, o registro de tarefas migrou do Excel (Figura 7), 


\section{2 - Congresso Brasileiro de Inovação e Gestão}

\section{de Desenvolvimento do Produto}

\section{1 a 13 de setembro de 2019 - Universidade de Brasília UnB}

utilizado durante a reunião, para o Trello, utilizado na rotina do dia-a-dia. Em seguida, os sprints se encerram com reuniões nas quais os entregues visuais eram avaliados e

\subsection{Análise da aplicação}

Descrita a aplicação conjunta do método Roadmapping e SCRUM, esse tópico busca efetuar análises sob perspectivas relacionadas à teoria e prática relacionadas à aplicação dos métodos expostos. Trata-se sobre o Roadmapping e SCRUM para aplicação da gestão ágil num ambiente de uma empresa Jr.

O método Roadmapping foi útil ao fornecer um framework visual estruturado integrando níveis distintos do processo de inovação, fornecendo suporte a todos eles. Especialmente em um ambiente no qual os envolvidos apresentavam pouca experiência em esforços de estratetização, se mostrou particularmente útil.

O SCRUM é um dos métodos que se destaca dentro da Gestão Ágil de Projetos. A aplicação desse método foi realizada com objetivo de adaptá-lo ao contexto de gestão de projetos em uma empresa Jr em crescimento. O procedimento exigiu a construção de um mapa com arquitetura adaptada e uma tabela no Excel. A aplicação do SCRUM foi iniciada com sucesso, no entanto, o acompanhamento em sequência a este artigo será essencial para garantir seu sucesso, bem como sua conexão com o roadmapping.

Percebeu-se, durante o processo, que agir sem um planejamento prévio dificultou o entendimento do processo como um todo, e assim, alguns aspectos poderiam ter sido feitos de forma diferente, com melhor resultado. A montagem do mapa foi um exemplo, pois ela foi feita sem se basear em qualquer estudo prévio e sem alguma experiência por parte dos alunos, apenas com a instrução do professor responsável. Caso tivesse sido feito o contrário, o mapa teria sido mais bem entendido e montado no primeiro momento, sem precisar de adaptações.

A aplicação do método na empresa pela Gerente de Projetos se atrasou por falta de conhecimento anterior à ação. Por isso, entende-se que ter realizado a pesquisa com uma base maior de conhecimento teria facilitado e adiantado o processo, além de realizado com mais entusiasmo e precisão pelos estudantes do GETI e pela equipe da Easy Consultoria Jr. Contudo, 


\section{2 - Congresso Brasileiro de Inovação e Gestão}

de Desenvolvimento do Produto

\section{1 a 13 de setembro de 2019 - Universidade de Brasília UnB}

o processo trabalho-aprendizado-retrabalho também foi um método de ensino, pois, no final, tornou-se possível que todos entendessem todo o processo ideal, baseando-se no processo real.

Para a empresa os métodos utilizados foram esclarecedores e facilitadores no processo de gestão de tarefas e projetos. A organização dos processos da empresa permitiu visualizar o que deve ser feito até o início do próximo ano, facilitando assim a distribuição de tarefas entre os membros da equipe e a cobrança dentro dos prazos determinados de acordo com a necessidade.

\section{CONCLUSÃO}

Este artigo teve por objetivo aplicar conjuntamente os métodos Roadmapping e SCRUM para ajudar na formulação e implementação da estratégia em uma empresa Jr., bem como fomentar atualizações rápidas e rotineiras da estratégia formulada, algo relevante para empresas pequenas.

A aplicação integrada dos métodos roadmapping-SCRUM favoreceu ao processo de planejamento estratégico e gestão de projetos da empresa Jr. analisada, podendo criar e visualizar de forma clara objetivos e benefícios para a mesma. A aplicação final dos dois métodos foi bem sucedida, embora considerações a respeito da falta de conhecimento prévio e ausência da etapa de planejamento do roadmap foram feitas, evidenciando que tais aspectos poderiam ter sido melhor trabalhados. Também será necessário um acompanhamento de maior prazo da aplicação do SCRUM, de modo a evidenciar sua real conexão com o roadmapping ao longo de todo o horizonte definido, isto é, um ano.

Logo, o recomendado seria ter iniciado o processo com um estudo profundo sobre os dois métodos e a relação entre os mesmos. No entanto, a abordagem aqui realizada pode ser compreendida como a melhor possível em um ambiente de urgência e escassez de recursos (como é típico em pequenas empresas). Propostas de estudo separadas da prática em tais ambientes podem ser vistas de modo negativo. A pesquisa ação foi útil ao auxiliar na conexão de ambos. 


\section{2ำ Congresso Brasileiro de Inovação e Gestão}

de Desenvolvimento do Produto

11 a 13 de setembro de 2019 - Universidade de Brasília UnB

Para que haja resultados mais robustos dos resultados da aplicação conjunta dos métodos, é crucial que haja maior prazo de acompanhamento da proposta aqui realizada. Isso terá o objetivo de garantir a realização de reuniões regulares com toda a equipe, para assim atualizar a aplicação dos métodos, realizando alterações caso apareçam impossibilidades na execução das metas planejadas.

\section{REFERÊNCIAS}

CARLOS, R.; AMARAL, D. C.; CAETANO, M.. Framework for continuous agile technology roadmap updating. Revista Innovation \& Management, v. 15, n. 3, pp. 321-336, 2018.

COUGHLAN, Paul; COGHLAN, David. Action research for operations management. International journal of operations \& production management, v. 22, n. 2, p. 220-240, 2002.

FREITAS, J.; MUDRIK, J.; de MELO, J.; BAGNO, R.; OLIVEIRA, M. On the combination of strategy and innovation tools with roadmapping: exploring taxonomies and sequences. International Association for Management of Technology (IAMOT). Conference Proceedings. Vienna, 2017.

GERDSRI, Nathasit; VATANANAN, Ronald S.; DANSAMASATID, Sasawat. Dealing with the dynamics of technology roadmapping implementation: A case study. Technological Forecasting and Social Change, v. 76, n. 1, p. 50-60, 2009.

KERR, Clive et al. Key principles for developing industrially relevant strategic technology management toolkits. Technological Forecasting and Social Change, v. 80, n. 6, p. 10501070, 2013.

OLIVEIRA, Maicon et al., Roadmapping: uma abordagem estratégica para o gerenciamento da inovação em produtos, serviços e tecnologias. Elsevier Brasil, 2012.

PHAAL, R.; FARRUKH, C.; PROBERT, D. Roadmapping for Strategy and Innovation: Aligning technology and markets in a dynamic world. University of Cambridge, UK: Institute of Manufacturing. 2010.

PHAAL, Robert; FARRUKH, Clare; PROBERT, David R. Fast-start roadmapping workshop approaches. In: Technology Roadmapping for Strategy and Innovation. Springer, Berlin, Heidelberg, 2013. p. 91-106.

PHAAL, Robert; FARRUKH, Clare; PROBERT, David. T-Plan: the fast-start to technology roadmapping: planning your route to success. University of Cambridge, Institute for Manufacturing, 2001. 
12 Congresso Brasileiro de Inovação e Gestão

de Desenvolvimento do Produto

11 a 13 de setembro de 2019 - Universidade de Brasília UnB

PHAAL, Robert; MULLER, Gerrit. An architectural framework for roadmapping: Towards visual strategy. Technological Forecasting and Social Change, v. 76, n. 1, p. 39-49, 2009.

SCHWABER, Ken. Agile project management with Scrum. Microsoft press, 2004.

SOUZA, M. L. P. Empreendedorismo Tecnológico: Processo de Geração de Startups (Pstart) e Métodos de Suporte ao Reconhecimento, Criação e Exploração de Oportunidades. 2018. 178 f. Trabalho de Conclusão de Mestrado (Mestre em Inovação Tecnológica e Propriedade Intelectual) - Universidade Federal de Minas Gerais, Belo Horizonte, 2018. [Orientador: Prof. Lin Chin Cheng].

SOUZA, M. L. P.; MELO FILHO, L. R.; OLIVEIRA, C. G. G.; ANICETO, M. D.; SILVEIRA, C. A.. Aplicação conjunta de métodos no desenvolvimento de startups: descrição e análise crítica. $1^{\circ}$ Congresso Brasileiro de Inovação e Gestão de Desenvolvimento do Produto. 2017.

SOUZA, Matheus L. P. et al. A Process Model Integrated to Innovation Management Tools to Support Technology Entrepreneurship. In: 2018 Portland International Conference on Management of Engineering and Technology (PICMET). IEEE, 2018. p. 1-12.

THIOLLENT, Michel. Pesquisa-ação nas organizações. Editora Atlas, 2009. 